\title{
Evaluation of Spatial Variation of Nonlinear Energy Transfer by Use of Turbulence Diagnostic Simulator ${ }^{*)}$
}

\author{
Naohiro KASUYA, Satoru SUGITA ${ }^{1)}$, Makoto SASAKI, Shigeru INAGAKI, Masatoshi YAGI'), \\ Kimitaka ITOH $^{1)}$ and Sanae-I. ITOH \\ Research Institute for Applied Mechanics, Kyushu University, Kasuga, Fukuoka 816-8580, Japan \\ ${ }^{1)}$ National Institute for Fusion Science, Toki, Gifu 509-5292, Japan \\ 2) Japan Atomic Energy Agency, Obuchi, Rokkasho-mura, Aomori 039-3212, Japan
}

(Received 6 December 2012 / Accepted 28 March 2013)

\begin{abstract}
Turbulence Diagnostic Simulator is an assembly of simulation codes to clarify the formation mechanism of turbulent structures by numerical diagnostics in magnetically confined plasmas. Global simulations are carried out using a reduced MHD model of drift-interchange mode in helical plasmas, and time series data of 3-D fluctuation fields are produced. It includes localized modes in their rational surfaces, and broad modes spread in the radial direction. Magnitudes of nonlinear couplings from the convective derivative are evaluated in the nonlinear saturated states. The radial profile shows that there exist strong mode excitation near the center, various modes and nonlinear couplings with higher $m$ modes in the middle radius, small number of propagating modes near the edge, which contribute to the pressure profile modification. For the detection of the different features, combination of several diagnostics is necessary.
\end{abstract}

(C) 2013 The Japan Society of Plasma Science and Nuclear Fusion Research

Keywords: turbulence simulation, structural formation, fluctuation, nonlinear coupling, drift-interchange mode, radial profile, non-local transport

DOI: $10.1585 /$ pfr.8.2403070

\section{Introduction}

It is important to clarify the role of turbulence on anomalous transport in toroidal plasmas [1]. Progress in experimental techniques enables to make quantitative estimation of turbulence transport with high resolution measurements of fluctuations [2]. Global simulations give long time series of three-dimensional turbulent fields, and analyses simulating experimental diagnostics on the fields can show how the characteristic feature is observed with consideration of physical mechanism and spatio-temporal resolution of the diagnostics [3]. This numerical diagnostic or synthetic analysis $[4,5]$ is useful for cross-validation between experiments and simulations. For that purpose, we have been developing Turbulence Diagnostic Simulator (TDS), which is the combination of fluid turbulence codes, measurement modules and analysis routines, to carry out numerical experiments of turbulent structural formation [6]. We have carried out analyses for a linear device, and clarified elementary processes by comparing with the experimental results $[7,8]$. In this paper, drift-interchange modes with a simplified helical plasma model are analyzed, using the TDS. Nonlinear energy transfer rate is evaluated to show multiple interactions of excited modes in the case with radially-spread modes, and the way for detection of the characteristic mechanism is discussed.

author'se-mail: kasuya@riam.kyushu-u.ac.jp

*) This article is based on the presentation at the 22nd International Toki Conference (ITC22).

\section{Model}

To provide turbulence data, a simulation code has been developed to calculate the drift-interchange turbulence in helical plasmas with a circular cross-section [3]. The set of model equations consists of charge conservation, parallel component of induction equation and sum of pressure evolution equations:

$$
\begin{aligned}
& \vec{\nabla} \cdot \vec{J}=0, \\
& \frac{\partial A}{\partial t}+E_{/ /}=\vec{B} \cdot \vec{\nabla} u, \\
& \frac{\partial P}{\partial t}+\sum_{s=\mathrm{i}, \mathrm{e}}\left[\left(\vec{v}_{s} \cdot \vec{\nabla}\right) P_{s}+\gamma P_{s} \vec{\nabla} \cdot \vec{v}_{s}\right]=0,
\end{aligned}
$$

where $\vec{J}$ is the current, $\vec{B}$ is the magnetic field, $u$ is the stream function, $A$ and $E_{/ /}$is the parallel component of the vector potential and electric field, respectively, and $\gamma$ is the specific heat ratio. $P_{s}$ and $\vec{v}_{s}$ are the pressure and velocity of each component, where $s=\mathrm{i}$ and e corresponds to a quantity of ions and electrons, respectively and $P$ is the sum of the pressure of ions and electrons. Here, $\vec{v}_{\mathrm{i} \perp}=\vec{v}_{\mathrm{e} \perp}=\vec{v}_{\perp}=\vec{E} \times \vec{B} / B^{2} \sim \vec{E} \times \hat{\zeta} / B_{0}$ is assumed for simplicity, and $\vec{v}_{\perp}=\vec{\nabla} u \times \hat{\zeta}$ leads $u=-\phi / B_{0}$, where $\vec{\zeta}$ denotes the unit vector in the toroidal direction and $B_{0}$ is the toroidal magnetic field of the leading order. Heat flux term is neglected in Eq. (3) for simplicity. The ordering and averaging method with the stellarator expansion [9] is applied to give a set of model equations for $u, A$ and $P$. Noted that orderings $v_{e / /}=O(\varepsilon)$ and $\nabla_{/ /} v_{\mathrm{e} / /}=O(\varepsilon)$ are used 
to include the parallel dynamics of electrons, where $\varepsilon$ is the inverse aspect ratio. In that case, the flow can be compressible, so the third term in the left hand side of Eq. (3) is kept in the model. Currents

$$
\overrightarrow{J_{\perp}}=-\left(n_{\mathrm{i}} m_{\mathrm{i}} \frac{\mathrm{d} \vec{v}_{\mathrm{i}}}{\mathrm{d} t}+\vec{\nabla}_{\perp} P\right) \times \frac{\vec{B}}{B^{2}},
$$

which is given from one-fluid equation of motion

$$
n_{\mathrm{i}} m_{\mathrm{i}} \frac{\mathrm{d} \vec{v}}{\mathrm{~d} t}=-\vec{\nabla} P+\vec{J} \times \vec{B},
$$

and

$$
\vec{J}_{/ /}=\sigma \vec{B}
$$

are substituted into Eq. (1) to give

$$
-\frac{n_{\mathrm{i}} m_{\mathrm{i}}}{B_{0}} \frac{\mathrm{d}}{\mathrm{d} t} \nabla_{\perp}^{2} u=\vec{B} \cdot \vec{\nabla} \sigma+\frac{\vec{\nabla} B^{2} \times \vec{\nabla} P}{B^{4}} \cdot \vec{B},
$$

where $\mu_{0} \sigma=-\nabla_{\perp}^{2} A / B_{0}, \mu_{0}$ is the permeability, $n_{\mathrm{i}}$ and $m_{\mathrm{i}}$ is the density and mass of ions, respectively. The parallel component of the electron equation of motion (Ohm's law) with charge neutrality condition $n_{\mathrm{i}}=n_{\mathrm{e}}=N$

$$
E_{/ /}=\eta J_{/ /}-\frac{\nabla_{/ /} P_{\mathrm{e}}}{N e}
$$

is substituted into Eq. (2) to give

$$
\frac{\partial A}{\partial t}+\eta J_{/ /}=\vec{B} \cdot \vec{\nabla}\left(u+\frac{P_{\mathrm{e}}}{N e B_{0}}\right),
$$

where $\eta$ is the resistivity. Equation (3) is reduced to

$$
\begin{aligned}
\frac{\partial P}{\partial t}+ & (\vec{\nabla} u \times \hat{\zeta}) \cdot \vec{\nabla}_{\perp} P+\gamma P \vec{\nabla}_{\perp} \cdot(\vec{\nabla} u \times \hat{\zeta}) \\
& -\gamma P_{\mathrm{e}} \nabla_{/ /} \frac{\nabla_{\perp}^{2} A}{\mu_{0} N e}=0 .
\end{aligned}
$$

In the helical plasmas, the external helical magnetic field produces the short-wavelength variation comparable to the helical pitch length, but a tractable model can be obtained by averaging the variation for the analysis of instability. The averaging procedure [9] is applied on Eqs. (7), (9) and (10) to give the following set of model equations:

$$
\begin{aligned}
& \frac{\partial \nabla_{\perp}^{2} u}{\partial t}=\left[u, \nabla_{\perp}^{2} u\right]+\nabla_{/ /} \nabla_{\perp}^{2} A+[\Omega, P]+\mu \nabla_{\perp}^{4} u, \\
& \frac{\partial A}{\partial t}=\nabla_{/ /}(u+\alpha P)+\eta \nabla_{\perp}^{2} A, \\
& \frac{\partial P}{\partial t}=[u, P]-C\left(\alpha \nabla_{/ /} \nabla_{\perp}^{2} A+[\Omega, u]\right)+\chi \nabla_{\perp}^{2} P+S,
\end{aligned}
$$

where $\nabla_{/ /}=\partial / \partial \bar{\zeta}+[, \Psi], \Psi=A-\Psi_{0}, \Psi_{0}=$ $-(1 / 2) \overline{\nabla\langle\Phi\rangle \times \nabla \Phi}, \quad \Phi$ is the magnetic potential, $\alpha=$ $V_{\mathrm{A}} /\left(2 \Omega_{\mathrm{ci}} a\right), C=\gamma \beta, \beta=P_{0} /\left(B_{0}^{2} / 2 \mu_{0}\right), S$ is the pressure source, $V_{\mathrm{A}}=B_{0} / \sqrt{\mu_{0} n_{\mathrm{i}} m_{\mathrm{i}}}$ is the Alfvén velocity, $\Omega_{\mathrm{ci}}$ is the ion cyclotron frequency, $a$ is the minor radius, $\mu$ is viscosity, $\chi$ is thermal conductivity, $\bar{F}$ is the average of $F$ over the helical pitch length, $\langle F\rangle$ is the integral of $F$ with respect to $\zeta$ whose integral constant is given by the condition \langle\rangle$=0$, and [ ] is the Poisson bracket. In this model, the helical magnetic field produced by the helical windings is given with $B_{h}=\nabla \Phi$. The total magnetic curvature $\Omega=2 r \cos \theta+\overline{(\nabla \Phi)^{2}}$ is composed of the toroidal (1st term) and helical (2nd term) curvatures. The toroidal magnetic field is inversely proportional to the major radius, so the toroidal curvature can be approximated to be proportional to the minor radius $r$. The helical field has the order of $\varepsilon^{1 / 2}$ in the stellarator expansion, so its square appears in this order. The following normalizations are used in the model equations: $u /\left(\varepsilon a V_{\mathrm{A}}\right) \rightarrow u, A /\left(\varepsilon a B_{0}\right) \rightarrow A, P /$ $\left(\varepsilon B_{0}^{2} / \mu_{0}\right) \rightarrow P, t / t_{\mathrm{A}} \rightarrow t$, and $r / a \rightarrow a$, where $t_{\mathrm{A}}=a / \varepsilon V_{\mathrm{A}}$ is the Alfvén time. The model is extended from that in Ref. [9] to include the parallel dynamics of electrons, and the magnetic curvature term in the pressure evolution equation to satisfy the energy conservation as

$$
\begin{aligned}
\frac{\partial}{\partial t}\left(E_{\varphi}+E_{A}+E_{p}\right) \\
=-\int\left[\mu\left(\nabla_{\perp}^{2} u\right)^{2}+\eta\left(\nabla_{\perp}^{2} A\right)^{2}+\chi\left(\nabla_{\perp} P\right)^{2}\right] \mathrm{d} r^{3} \\
\quad+\int P S \mathrm{~d} r^{3},
\end{aligned}
$$

where $E_{\varphi}=\int \mathrm{d} r^{3}\left(\nabla_{\perp} u\right)^{2} / 2, E_{A}=\int \mathrm{d} r^{3}\left(\nabla_{\perp} A\right)^{2} / 2$ and $E_{p}=\int \mathrm{d} r^{3} P^{2} /(2 C)$. Coefficient $C$ has dependency on the pressure, so $P^{2} / C$ can be considered to be the quantity related to the energy.

By setting parameters $\alpha$ and $C$ to be zero, the model reproduces the resistive interchange mode, which is localized in the rational surface. For nonlinear simulations in this paper, the magnetic curvature term in the pressure evolution equation is omitted to obtain more dynamical saturated states, whose time series data is used as target data for numerical diagnostics. Nonlinear terms that include $\alpha$ or $C$ are also omitted for simplicity. This model is used as the fundamental one not for electrostatic turbulence, but for including MHD modes, oscillatory modes and their nonlinear coupling.

Equations (11-13) are solved in the toroidal coordinates with the spectral expansion in the poloidal and toroidal directions. The boundary conditions in the radial direction are set to $F=0$ at $r=0,1$ when $m \neq 0$, and $\partial F / \partial r=0$ at $r=0, F=0$ at $r=1$ when $m=0$, where $F$ implies $\{u, A, P\}, m$ is the poloidal mode number, and $r=$ 1 gives the outer boundary of the plasma.

\section{Dynamical Saturation}

A nonlinear simulation is performed, using the following parameters: magnetic field $B_{0}=2.0$ [T], density $N=$ $1 \times 10^{19}\left[\mathrm{~m}^{-3}\right]$, beta ration $\beta=0.03$, minor radius $a=$ 0.6 [m], major radius $R_{0}=3.75[\mathrm{~m}]$ and specific heat ratio $\gamma=5 / 3$. These parameters gives $\alpha=0.06$ and $C=0.05$. The pressure source is fixed to be 


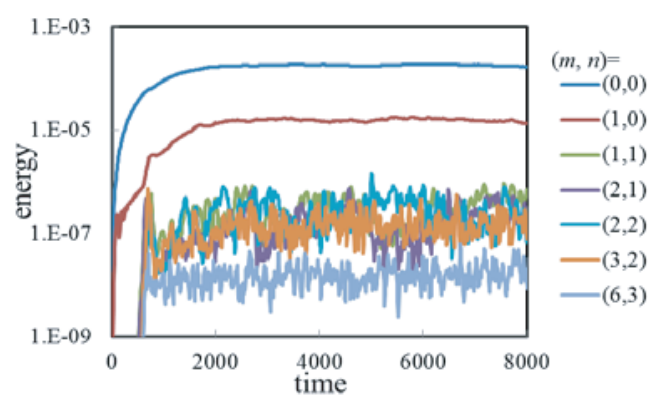

Fig. 1 Time evolutions of the energy of Fourier modes. The mean and fluctuating internal energies are shown.

$$
S(r)=\frac{4 S_{0 \chi}}{L_{\mathrm{N}}^{2}}\left[1-\left(\frac{r}{L_{\mathrm{N}}}\right)^{2}\right] \exp \left[-\left(\frac{r}{L_{\mathrm{N}}}\right)^{2}\right],
$$

with $S_{0}=0.15, L_{\mathrm{N}}=0.6[\mathrm{~m}]$, which forms the profile peaked at $r=0$. The magnetic potential is given by

$$
\Phi=2 \Phi_{l} I_{l}(h r) \sin (l \theta+h \zeta),
$$

where $I_{l}$ is the modified Bessel function, $l$ is the pole number of the helical winding, $h=M / R_{0}, M$ is the pitch number and $\Phi_{l}$ is a constant coefficient. In this case, $l=2, M$ $=10$ and $\Phi_{l}=0.71$ are used, which give rotational transform $\iota$ to be a monotonically increasing function with the radius from $\iota(0)=0.51$ to $\iota(a)=1.5$, so rational surfaces with $m / n=1 / 1$ and $3 / 2$ are included, but $2 / 1$ is not in the plasma, for example, where $m$ and $n$ are the poloidal and toroidal mode number, respectively. Coefficients $\mu, \eta$ and $\chi$ are set to $1 \times 10^{-4}$ as numerical viscosities. The differential equations in the radial direction are solved with 1024 radial grids, and Fourier modes $-32 \leq m \leq 32,-8 \leq n \leq 8$ are taken.

Spatio-temporal data of turbulent fields are generated by this global simulation. Figure 1 shows the time evolutions of the fluctuating internal energy $E_{p m n}=$ $\int \mathrm{d} r^{3} P_{m n}^{2} /(2 C)$. The simulation begins with a flat pressure profile in the initial state, and the flux-surface averaged pressure $((m, n)=(0,0)$ component $)$ increases by the pressure source. At $t \sim 200$, nonlinear terms drive the growth of $n=1$ modes. Low $m, n$ modes whose rational surfaces exist in the plasma become unstable after $t \sim 300$, and nonlinear saturation is obtained in $t \sim 800$. In the saturated state, mode structures of low $m, n$ modes, such as $(m, n)=$ $(1,1)$ and $(2,1)$, spread broadly in the radial direction, and those of medium $m, n$ modes, such as $(3,2)$ and $(5,5)$, are localized near their rational surfaces, as shown in Fig. 2. With the existence of these localized modes, flattening of the mean profile occurs at the rational surfaces as shown in Fig. 2 (c). The snapshot of the pressure fluctuation is shown in Fig. 3. There exist various sizes of vortexes in the poloidal cross-section, which rotate in the poloidal direction and are mixed with each other. Figure 4 shows the radial profile of the poloidal mode number spectrum and the frequency spectrum. There are low $m$ modes spread

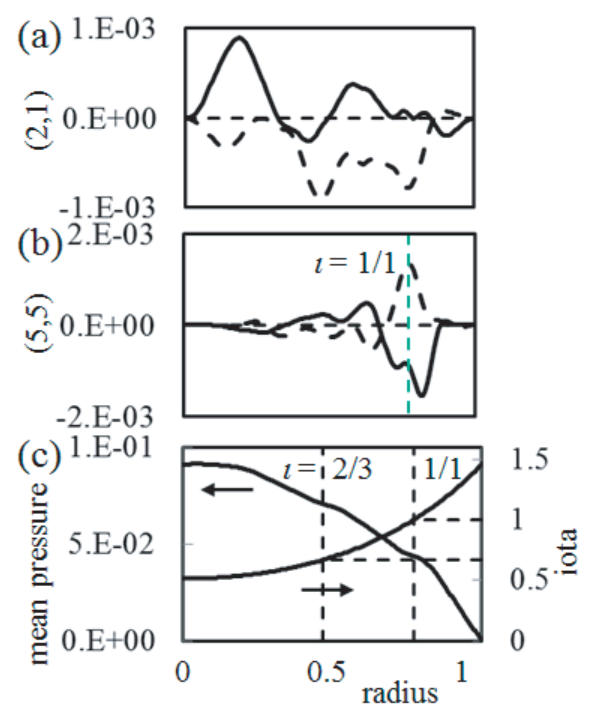

Fig. 2 Snapshots of eigenfunction of (a) the $(2,1)$ mode and (b) $(5,5)$ mode of the pressure at $t=6000$, where solid and dashed lines represent the real and imaginary part, respectively. The vertical line in (b) represents the position where the rational surface $1 / 1$ exists. (c) Mean pressure profile at $t=6000$ and rotational transform profile.

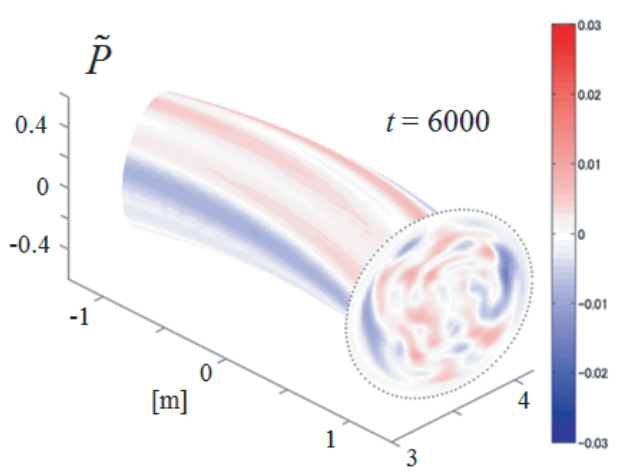

Fig. 3 Snapshots of 3-D structure of the pressure fluctuation at $t$ $=6000$.
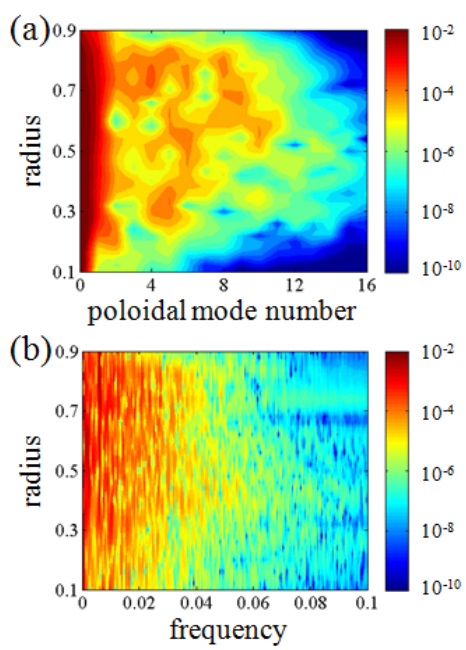

Fig. 4 Radial profile of (a) the poloidal mode number spectrum and (b) frequency spectrum of $P$. 
in the radial direction, so mutual couplings with several modes are possible, due to the overlapping of the modes, though these modes have no characteristic frequency.

The $\phi_{00}$ component, whose radial variation gives a mean poloidal flow, is also produced in the saturated state. The $\phi_{00}$ component has a characteristic frequency $f \sim$ 0.02 , and its radial structure is rather global, though there exists perturbations with radial mode number $K_{r}=3-9$ in some moments.

\section{Nonlinear Energy Transfer}

The contributions to energy evolutions from nonlinear couplings are evaluated in the saturated states to understand the spatial variation of the structural formation mechanism.

As for the pressure fluctuations, the evolution equation of the internal energy includes the contributions from the linear term (LT, 1st term in the right hand side of the following equation) and nonlinear terms from the convective derivative (NT1, 2nd term):

$$
\begin{aligned}
& \frac{\partial E_{P m n}}{\partial t}=\frac{1}{2 C} \int \mathrm{d} r^{3} P^{*} \\
& \quad \times\left\{\left(\frac{\partial u_{0}}{\partial r} \frac{\partial P}{r \partial \theta}-\frac{\partial P_{0}}{\partial r} \frac{\partial u}{r \partial \theta}-\alpha C \frac{\partial \nabla_{\perp}^{2} A}{\partial \zeta}+\alpha C \frac{\partial \Psi_{0}}{\partial r} \frac{\partial \nabla_{\perp}^{2} A}{r \partial \theta}\right)_{m n}\right. \\
& \left.\quad+[u, P]_{m n}+S_{m n}\right\} .
\end{aligned}
$$

Figure 5 shows the radial profile of the nonlinear contribution to formation of the $(m, n)=(0,0)$ component of the pressure $\left(P_{00}\right)$, where NT1 is decomposed into one with each poloidal mode number. In red regions, the $P_{00}$ component gets the energy from modes with poloidal mode number $m$, and in blue regions, vice versa. The radial profile of NT1 indicates the characteristic regions; $r / a<0.3$ : strong mode excitation, 0.4 - 0.7: existence of various modes, > 0.8: existence of small number of dominant modes, corresponding to the different features shown in the spectrums in Fig. 4 (a). The dominant mode to contribute to the $P_{00}$ component changes according to the radial position, as indicated by the marks in Fig. 5 (a). There are low $m$ modes $(1 \leq m \leq 3)$ widely spreading in the radial direction (Fig. 5 (b) 1)), which contributes to the nonlinear structural formation, especially in $r / a<0.3$. In addition, there is a radial region where nonlinear couplings with higher $m$ modes contribute to the pressure profile modification in $r / a=0.5-0.7$ (Fig. 5 (b) 2)). The profile modification arises in this region, and propagates to the other regions.

As for the flow generation, the evolution equation of the electrostatic potential energy includes the contributions from the linear term (LT, 1st term in the right hand side of the following equation), ballooning term to give a linear coupling with neighboring modes (TC, 2nd term), nonlinear terms from the convective derivative (NT1, 3rd term)
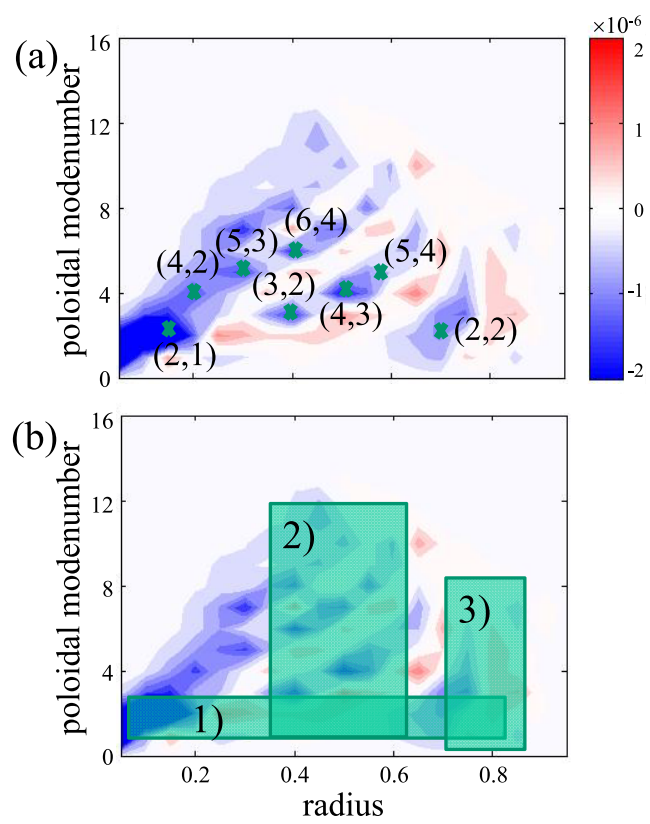

Fig. 5 Radial profile of the nonlinear contribution to the $P_{00}$ evolution, which is decomposed into each poloidal mode number component. (a) Mode numbers of the main contributors on each peak and (b) characteristic regions of the nonlinear couplings are also shown.

the parallel derivative (NT2, 4th term):

$$
\begin{aligned}
& \frac{\partial E_{\varphi m n}}{\partial t}=-\int \mathrm{d} r^{3} u^{*} \\
& \quad \times\left\{\left(\frac{\partial u_{0}}{\partial r} \frac{\partial \nabla_{\perp}^{2} u}{r \partial \theta}-\frac{\partial \nabla_{\perp}^{2} u_{0}}{\partial r} \frac{\partial u}{r \partial \theta}+\frac{\partial \nabla_{\perp}^{2} A}{\partial \zeta}-\frac{\partial \Psi_{0}}{\partial r} \frac{\partial \nabla_{\perp}^{2} A}{r \partial \theta}+\frac{\partial \Omega_{0}}{\partial r} \frac{\partial P}{r \partial \theta}\right)_{m n}\right. \\
& \left.\quad+\left(2 \cos \theta \frac{\partial P}{r \partial \theta}+2 \sin \theta \frac{\partial P}{\partial r}\right)_{m n}+\left[u, \nabla_{\perp}^{2} u\right]_{m n}+\left[\nabla_{\perp}^{2} A, A\right]_{m n}\right\} .
\end{aligned}
$$

NT2 comes from electromagnetic fluctuations in the $\nabla_{/ /} J$ term. The volume integration of each term shows that TC is the largest, so the main cause of the $\phi_{00}$ oscillation is the coupling between $\phi_{00}$ and $P_{ \pm 10}$.

For $P_{10}$, competition between negative LT and positive NT1 determines the evolution, so both the profile modification, which contributes to the variation of LT, and the nonlinear couplings, which contribute to NT1, must be taken into account. As is shown in Fig. 6, there is a region where the contributions from modes with higher $m$ mode numbers are strong in $r / a=0.5-0.8$. The region is not the same with Fig. 5 (a), depending on the effective 3-wave couplings.

For $\phi_{32}$, which has large amplitude, LT+TC and $\mathrm{NT} 1+\mathrm{NT} 2$ are comparable. Among the nonlinear contributions, NT2 is larger than NT1, and bursts of NT2 affect the evolution. Term NT2 is the contribution from $A$ variations, whose energy evolution equation includes the contributions from the linear term (LT, 1st term in the right hand side of the following equation) and nonlinear terms from 


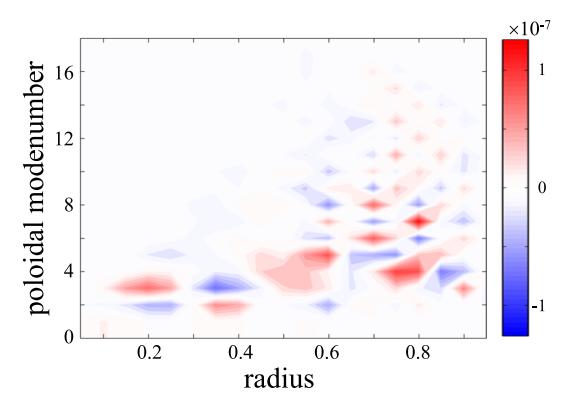

Fig. 6 Radial profile of the nonlinear contribution to the $P_{10}$ evolution.

the convective derivative (NT1, 2nd term):

$$
\begin{aligned}
& \frac{\partial E_{A m n}}{\partial t}=-\int \mathrm{d} r^{3} \nabla_{\perp}^{2} A^{*} \\
& \quad \times\left\{\left(\frac{\partial\left(u_{0}+\alpha P_{0}\right)}{\partial r} \frac{\partial A}{r \partial \theta}+\frac{\partial(u+\alpha P)}{\partial \zeta}-\frac{\partial \Psi_{0}}{\partial r} \frac{\partial(u+\alpha P)}{r \partial \theta}\right)_{m n}\right. \\
& \left.\quad+[u, A]_{m n}\right\} .
\end{aligned}
$$

\section{Summary and Discussion}

Here we analyze the spatial variation of nonlinear coupling to evaluate the non-local behavior in a turbulent plasma. Global simulations are carried out using a reduced MHD model of drift-interchange mode in helical plasmas. Time series data of 3-D fluctuation fields are obtained, and the nonlinear mechanism with existence both of localized modes and radially-spread modes is analyzed by calculating the nonlinear contributions to the energy evolution. The dominant mode changes according to the radial position. It is shown that low $m$ modes widely spreading in the radial direction contributes to the structural formation near the center, and the nonlinear couplings with higher $m$ modes in the middle radius contribute to the pressure profile modification. As for the flow generation, the toroidal coupling induces the potential oscillation.

The phenomena described in this simulation is the case with dynamical coupling of modes excited at each rational surface, and widely spread in the radial direction, which gives large correlation between separated radial positions. The radial profiles of nonlinear contribution, as shown in Figs. 5 and 6, are instructive for the experimental detection of this kind of phenomena. For the detection, combination of several diagnostics is necessary. There are three characteristic regions as shown in Fig. 5 (b). In the region 1 , there exists widely spread low $m$ mode, which connects the core and edge, so the measurement covering wide range of radius, such as the electron cyclotron emission measurement is suitable [10]. In the region 2 in the middle radius, couplings in wide range of wave number are important, so 2-D fine structure measurement, such as beam emission spectroscopy and multi-channel heavy ion beam probe [11], is necessary. In region 3, there are propagating modes, and reflectometor or electrostatic probes can be applied for this region near the edge [12]. Therefore, measurements covering the wide spatial range and localized measurements with high resolution can identify the nonlinear mechanism. We also carried out simulations of diagnostics, such as the heavy ion beam probe [3] and the beam emission spectroscopy [13]. In these simulations, effects of the finite spatial resolution are estimated to deepen our knowledge on what is observed with the experimental diagnostics.

Response to active control with additional modulation, such as by pellet injection [14] and electron cyclotron heating [10], is studied in experiments to clarify the nonlocal effect in plasma transport. Motivated by these experiments, we carried out the simulation to show the response to the pressure source modulation to clarify the role of each mode. By adding the periodic source modulation near the center, and extract the typical response with conditional averaging, the nonlinear dynamics described in this paper can be illuminated. The result will be reported in the separated article.

In this way, turbulence analysis using simulation data can give the insight for the physical mechanisms in plasmas. Common methods can be applied to various simulation data for the cross-validation using the TDS.

\section{Acknowledgments}

Authors acknowledge discussions with Prof. A. Fujisawa, Prof. K. Ida, Prof. A. Fukuyama, and Dr. Y. Nagashima. This work is supported by the Grant-in-Aid for Young Scientists (24760704) and for Scientific Research (23244113, 21224014) of JSPS, by the collaboration program of NIFS (NIFS11KNST013, NIFS12KNTT012) and of RIAM of Kyushu University.

[1] See reviews, e.g. P.H. Diamond et al., Plasma Phys. Control. Fusion 47, R35 (2005).

[2] See reviews, e.g. A. Fujisawa, Nucl. Fusion 49, 013001 (2009).

[3] N. Kasuya et al., Plasma Sci. Technol. 13, 326 (2011).

[4] C. Holland et al., Phys. Plasmas 16, 052301 (2009).

[5] L. Lin et al., Phys. Plasmas 16, 012502 (2009).

[6] N. Kasuya et al., Proc. 23rd IAEA Fusion Energy Conference, Daejeon, Korea (2010) THC/P4-15.

[7] T. Yamada et al., Nature Phys. 4, 721 (2008).

[8] N. Kasuya, M. Yagi, S.-I. Itoh and K. Itoh, Phys. Plasmas 15, 052302 (2008).

[9] M. Wakatani, Stellarator and Heliotron Devices (Oxford University Press, Oxford 1998).

[10] S. Inagaki et al., Phys. Rev. Lett. 107, 115001 (2011).

[11] A. Fujisawa et al., Phys. Rev. Lett. 93, 165002 (2004).

[12] Y. Nagashima et al., Phys. Rev. Lett. 95, 095002 (2005).

[13] N. Kasuya et al., Proc. 38th EPS Conf. on Plasma Phys., Strasbourg, 2011, P4.113.

[14] N. Tamura et al., Nucl. Fusion 47, 449 (2007). 